HENRI FRANÇAIS, DE QUELQUÉS PERFECTIONNEMENTS ETC. I67

\title{
DE QUELQUES PERFECTIONNEMENTS RÉCENTS DANS LES MÉTHODES D'EXAMEN DU LIQUIDE CÉPHALO-RACHIDIEN
}

PAR LE

DR. HENRI FRANÇAIS, ancien chef de clinique à la Salpêtrière.

On sait tout l'intérêt que présente l'étude du liquide céphalo-rachidien pour le diagnostic des affections du système nerveux. Dans l'étude des syndromes épileptiques en particulier, l'examen de ce liquide, pratiqué avec soin, permet, dans certains cas, de déterminer la nature de l'affection causale, et d'instituer, à temps, une therapeutique appropriée. Aussi, ne doit-on négliger aucun des procédès d'étude propres à mieux faire connaitre ce liquide et à mieux appréciér les réactions chimiques et biologiques dont il est le siège. Mon but n'est pas de décrire ici la technique de la ponction lombaire, ni les procédés classiques et courants d'examen du liquide céphalo-rachidien. Je désire insister seulement sur certaines notions d'acquisition récente. Ce sont: la mesure de la tension intra-cranienne, l'étude chimique du liquide, et enfin son examen cytologique, et biologique.

Le syndrome de l'hypertension cranienne se manifeste par des symptômes souvent très variés dans leur expression clinique. Aussi la recherche de la pression a-t-elle pris une grande valeur depuis que les travaux modernes ont signalé l'importance des variations de la tension intracranienne. De nombreux appareils ont été proposés pour effectuer cette mesure. Quincke se servait d'un manomètre à air libre. M. H. Claude a fait construire un petit manomètre anérö̈de soigneusement gradué en centimètres d'eau que l'on peut relier directement au moyen d'un tube à caoutchouc à un dispositif de robinet à trois voies. Ce dernier, suivant qu'il occupe l'une ou l'autre position, fait 
communiquer l'aiguille avec l'extérieur ou avec le manomètre. Chez les sujets normaux, la pression est, d'après M. Claude, de Io à ${ }_{5}$ centimètres d'eau, dans la position couchée. La position assise élève, de 8 à Io centimètres, la pression. Le chiffre le plus faible de pressions constatées a été de 4 centimètres. Les chiffres les plus élevés ont varié de $3^{8}$ à 62 . Fréquemment, dans les tumeurs céré. brales, et dans la plupart des affections génératrices d'épilepsie, on trouve de 40 à 45 . Sauf dans les cas ou une néoformation méningée exclut les ventricules cérébraux de la grande cavité arachnoïdo-pie-mérienne, on aura, grâce à ce manomètre, un moyen pratique de constater l'existence de l'hypertension intra-cranienne et d'en mesurer le degré.

L'étude chimique du liquide céphalo-rachidien présente des particularités dignes d'être mentionnées. On sait que le liquide céphalo-rachidien renferme, à l'état normal, de l'albumine, des chlorures, du sucre et de l'urée. Seules, les quantités d'albumine et de chlorures sont intéressantes à connaître au point de vue pratique. Pour doser les chlorures, on emploie la solution à $29 \mathrm{gr}, 07$ d'azotate d'argent par litre, étendue au cinquième et renfermée dans un tube gradué en centimètres. On met 2 centimètres cubes du liquide à doser avec 2 gouttes de chromate de potasse, on verse la solution de nitrate d'argent, jusqu'à coloration orangée persistante, on lit le nombre de graduations employées et on a ainsi le titrage des chlorures en grammes. L'abaissement des chlorures est synonyme d'inflammation méningée.

L'albumine céphalo-rachidienne peut se rechercher à l'aide de la chaleur seule, de la chaleur et de l'acide acétique, du sulfate d'ammoniaque, du réactif de Tanret, de l'acide nitrique à froid. On peut aussi essayer de doser cette albumine à l'aide de pesées et d'échelles comparatives en constituant une gamme étalon avec plusieurs tubes témoins, renfermant un liquide opalescent dont la teinte plus ou moins accusée équivant à des pourcentages variables du taux de l'albumine. Lorsqu'on veut simplement constater 
DE QUELQUES PERFECTIONNEMENTS RÉCENTS ETC. $\quad 169$

la présence d'albumine, il suffit de la rechercher par la méthode de l'acide nitrique à froid.

Voici la technique conseillee par M. Sicard: A deux centimètres cubes environ de liquide céphalo-rachidien recueilli dans un tube à essai, de dimension relativement faible, il ajoute à l'aide d'un flacon compte-gouttes 6 à 7 gouttes d'acide nitrique nitreux qu'il fait tomber sur la paroi de tube à quelque distance seulement du niveau du liquide. Un précipité se forme. Il convient d'attacher une grande valeur à l'apparition immédiate du couche albumineux dès l'arrivée des trois ou quatre premières gouttes d'acide nitrique au contact du liquide. Il faut tenir compte aussi de l'intensité de la réaction après une attente de deux à trois minutes. Dans ces conditions, les troubles ainsi déterminés peuvent varier de l'opalescence au trouble epais. L'opalescence simple se rencontre dans tout liquide cephalo-rachidien normal.

L'hyperalbuminose, s'accompagnantgénéralement d'hyperleucocytose, est synonyme de lésion organique. Plus le taux de l'albumine est élevé, plus l'affection a des chances d'être grave. D'après Mestrezat: 8 à Io grammes d'albumine, dans un cas chronique, doivent faire penser au syndrome de coagulation massive. Dans les méningites aigues, aux environs de I gr., il s'agit d'une affection atténuée, d'une méningite fruste cérébro-spinale ou autre. Jusqu'à 3 grammes, il faut penser à la méningite tuberculeuse et s'aider de la recherche des chlorures. Au de là de 3 grammes, il $\mathrm{y}$ a possibilité de méningite cérébrospinale ou pneumococcique. Dans les méningites chroniques, la valeur diagnostique est moins grande. La quantité permet seulement de juger de l'importance de l'atteinte méningée, et de son allure torpide ou aigue. Au cours des infections générales, l'augmentation d'albumine montre l'atteinte méningée.

En I9ro, Sicard et Foix ont attire l'attention sur les réactions albumineuses du liquide céphalo-rachidien au cours des pachyméningites rachidiennes et ont isolé un 
syndrome humoral qu'ils ont appelé syndrome de dissociation albumino-cytologiqué. Celui-ci est caractérisé par une disproportion manifeste entre la quantité d'albumine et la réaction cytologique, dissociation des élements chimiques et cellulaires qui s'affirme par l'intensité de la réaction albumineuse et la pénurie ou l'absence de la réaction lymphocytaire. Ce phénomène peut se trouver associé à la xantochromie du liquide, à de l'albuminose et à de l'hypoglycose rachidienne. Vers la même époque, j'ai signalé avec M. Baudouin l'absence de parallélisme entre la présence de la lymphocytose et celle de l'albumine dans le liquide céphalo-rachidien. La constatation de ce signe a une grande importance diagnostique lorsqu'il s'agit d'interprêter certains syndromes sensitifs ou sensitivo-moteurs des membres. II permet quelquefois de soupçonner, avant tout autre symptòme, un début de compression de la moëlle. M. Sicard a emis l'hypothèse que cette réaction dissociée était fonction de la compression des veines péri-dure-mériennes épidurales et des trous de conjugaison. Il s'agirait là d'un processus de stase veineuse et d'une transsudation du plasma sanguin.

De tous les examens qui portent sur le liquide céphalorachidien, celui qui, de beaucoup, fournit les renseignements les plus précieux est incontestablement l'examen cytologique. Il se pratique à l'aide de la cellule Nageotte et de la centrifugation.

La cellule de Nageotte comprend une lame porte-objet sur laquelle se trouve une cellule d'un millimètre de profondeur. Le fond dont la superficie est d'un centimètre carré est divisé en une série de raies parallèles, distantes d'un quart de millimètre. Le liquide céphalo-rachidien, recueilli immédiatement après ponction et sans centrifugation préalable, est, après addition d'une ou deux gouttes de bleu polychrome, disposé dans la cellule. On recouvre avec une lamelle, on laisse reposer quelques minutes, et on compte, sous le microscope, les élements contenus dans plusieurs divisions. La contenance d'une division 
étant de $\mathrm{x}$ millimètre cube, 25 , il suffit de diviser par $\mathrm{I}, 25$ le nombre moyen d'éléments qui y sont contenus, pour connaître la nombre d'éléments par millimètre cube.

La numération des éléments du liquide céphalo-rachidien dans la cellule de Nageotte est un procédé des plus simples et d'une grande précision. Mais il est insuffisant pour établir une formule hémoleucocytaire. Il est nécessaire, pour cela, de recourir à la centrifugation afin de séparer les élements figurés du liquide qui les contient. La méthode classique, ordinairement employée pour le cyto-diagnostic est passible de divers reproches. Tel qu'il a été réglé par MM. Widal et Ravaut, l'examen cytologique expose à de grosses altérations cellulaires qui tiennent, pour la plupart, à ce fait qu'on laisse déssécher, à l'air libre, ou à l'étuve, la goutte de liquide renfermant les eléments. Pour le cas particulier du liquide céphalo-rachidien, M. Alzheimer a beaucolp insisté sur les défectuosités de cette technique, au point de vue de l'examen cytologique. Convaincu, ainsi que que M. Nissl, de l'importance que pourrait avoir une bonne conservation des éléments dans certains cas de diagnostic neurologique, M. Alzheimer ${ }^{1}$ ) a proposé de remplacer la methode française par un procédé beaucoup plus compliqué. Il consiste à mêler le liquide céphalo-rachidien avec un fixateur approprié (ordinairement l'alcool) dans un tube à centrifuger. Après centrifugation, les éléments cellulaires, ordinairement enrobés dans de l'albumine coagulée, forment un petit caillot qui est inclus dans la celloïdine ou la paraffine et coupé en série. Cette méthode donnerait d'excellents résultats. M. Alzheimer insiste sur ce fait que les cellules $\mathrm{du}$ liquide cephalo-rachidien normal ou pathologique sont parfaitement conservées, et que leur aspect ne ressemble en rien à ce que donnent les méthodes ordinaires. Quels que soient les avantages de la méthode d'Alzheimer, il est certain que l'on ne saurait la considerer que comme un

1) Alzheimer. - Einige Methoden zur Fixierung der zelligen Elemente der Cerebrospinalflussigkeit (Zentralblatt för Nervenheilkunde und Psych., I5. Juni r907, S. 449. 
procédé d'exception en raison de sa complexité. C'ést pourquoi, M. Baudouin ${ }^{1}$ ) et moi, avons proposé un autre procédé permettant d'obtenir de bonnes préparations cytologiques. Nous employons un tube à centrifuger à fond plat et mobile. Ce fond est constitué par une lamelle circulaire qui est appliqué elle-même sur un disque de verre bien dressé avec lequel elle fait corps. C'est sur cette lamelle que la centrifugation applique directement les éléments qui seront secondairement fixés et colorés. Les manipulations sont donc réduites à leur minimum, les éléments étant d'emblée fixés sur la lamelle qui sera, à son tour, après coloration, mise sur une lame, comme on le fait dans la méthode classique d'examen des microbes sur lamelle. On obtient, de la sorte, une préparation permanente que l'on peut conserver sans plus de difficulté qu'une preparation ordinaire.

(Suite et fin dans le prochain numéro.)

\section{TRAITEMENT DE L'ÉPILEPSIE SANS BROMURES \\ PAR LE Docteur CÉSAR JUARROS, de Madrid.}

Communication présentée au $\mathrm{V}$ Congrés International pour l'assistance des aliénés tenu à Moscou.

\section{OPINIONS SUR L'ÉPILEPSIE.}

Une faute d'origine s'oppose à la solution du problème de l'épilepsie. Cette faute est due à la confusion de l'épilepsie avec l'attaque épileptique. La crise convulsive est une manifestation clinique des plus frappantes de l'épilepsie; mais ce n'est pas l'épilepsie.

L'épilepsie, suivant mon insistance depuis quelques

1) A. Baudouin et $H$. Francais. - Un nouveau tube à centrifuger (tube à fond plat et mobile). (Soc. de biologie, I5 février rgra) et Paris médical, to janvier 19r4. 